\title{
An Elementary Algorithm for Digital Line Recognition in the General Case
}

\author{
Lilian Buzer \\ A2SI Laboratory, ESIEE, 2 bd Blaise Pascal, \\ Cité Descartes, - BP 99, 93162 Noisy-Le-Grand Cedex, France \\ Buzerl@esiee.fr \\ Institut Gaspard Monge, Unité Mixte CNRS-ESIEE, UMR 8049
}

\begin{abstract}
This paper is concerned with the naive and, more generally, $\alpha$-thick digital line recognition problem. Previous incremental algorithms deal with the 8-connected case [DR95] or with sophisticated machinery coming from Linear Programming [Buz03]. We present the first elementary method [Buz02] that works with any set of points (not necessarily 8-connected) and we propose a linear time algorithm under some restrictions (which were implicitly assumed in [DR95]). This paper deals with implementation details giving pseudo-code of our method. We insist on linking the recognition problem to the intrinsic properties of convex hulls.
\end{abstract}

Keywords: Digital line, incremental, recognition, convex hull, thickness, implementation.

\section{Introduction}

When one processes digital images, one also sometimes wants to know how to recognize basic geometric entities. In this way appears the recognition problem of digital lines of variable thickness (see Fig. 1). In this paper, we set up the definition of $\alpha$-thick digital line which allows to represent a wide variety of digital line segments. We show that the knowledge of some basic information on the convex hull of a set of points $S$ (thickness or critical supporting lines) is sufficient to determine if $S$ is a subset of an $\alpha$-thick digital line segment. Then, we adapt this remark in order to create an elementary algorithm for the recognition problem. We exhibit its optimal complexity relatively to many different configurations (static, incremental, dynamic). Thus, we propose a short implementation of the linear incremental version which works when points are inserted in a given direction. This new method and the previous one based on Megiddo algorithm [Buz03] are able to incrementaly process $\alpha$-thick digital line with non-connected pixels. In [Buz03] we only deal with naive digital line (where $\alpha=1$ ), but this previous method is able to recognize $\alpha$-thick digital line by replacing the thickness of 1 by $\alpha$. Conversely, the new approach can operate naive digital line when we set $\alpha$ equal to 1 . If the two algorithms recognize the same objects using the same thickness notion, the way they work is completely 
different. The Megiddo approach does not explicitly compute the new thickness value when a point is inserted. We can only know if the current thickness is below a given threshold. With the new method, we are able to determine the current thickness value at any moment and to add other criteria that can be estimated from the thickness : density of pixels or curvature. This entails a higher complexity $O(n \log n)$ but in most cases we will work in configurations that allow to use a linear and elementary version of this new approach. Throughout this paper, we will denote by $O_{x}$ and $O_{y}$ the axes of the Cartesian coordinate system, by $e_{x}$ and $e_{y}$ the associated units vectors and by $(x, y)$ the Cartesian components of points.

\section{$2 \quad \alpha$-Thick Digital Line}

We hereafter present $\alpha$-thick digital line. Its seminal definition was given by Reveillès in [Rev91]. Any digital line $D$ in $\mathbb{Z}^{2}$ is described by a set of parameters: the normal vector $N=(a, b)$ in $\mathbb{Z}^{2} \backslash\{0\}$ with $\operatorname{gcd}(a, b)$ equal to 1 , the inferior bound $\gamma$ in $\mathbb{Z}$ and the arithmetic thickness $w$ in $\mathbb{Z}$. A point $(x, y)$ of a digital line with parameters $(a, b), \gamma, w$ verifies the following diophantine inequality:

$$
\gamma \leq a . x+b . y<\gamma+w
$$

If we choose the arithmetic thickness to be equal to the infinity norm of the normal vector: $\|N\|_{\infty}=\sup \{|a|,|b|\}$, we obtain an 8-connected object called $a$ naive digital line (see Fig. 10. We extend this definition to the $\alpha$-thick digital line where $\alpha$ corresponds to a thickness ratio whose reference $(\alpha=1)$ is the naive digital line. A point $(x, y)$ belongs to such a line if it verifies:

$$
\gamma \leq a . x+b . y<\gamma+\alpha \cdot \sup \{|a|,|b|\}
$$

We can consider three different subclasses of digital lines depending on the components of the normal vector $N=(a, b)$. When $|a|=|b|=1$, the digital line has a slope of $\pm 45^{\circ}$. When $|a|<|b|$ (resp. $|b|<|a|$ ), the resulting slope relative to the $O_{x}$ axis (resp. $O_{y}$ axis) is always comprised between $-45^{\circ}$ and $+45^{\circ}$ (see Fig. 2).

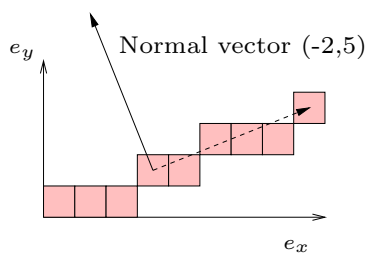

(a) a naive digital line

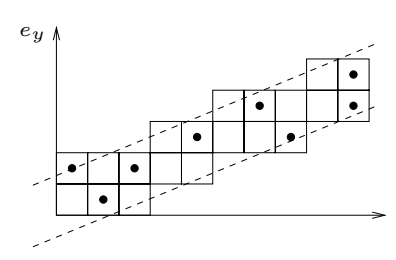

(b) 2-thick digital line covers $P$

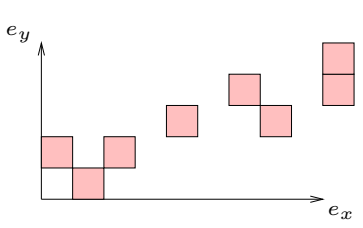

(c) a given set of points $P$

Fig. 1. The recognition problem using different thickness for the digital lines 


\section{Thickness Criterion}

\subsection{Introducing the Notion of Thickness}

The definition of a digital line is intrinsically algebraic. We use an equivalent characterization which is more linked to the field of Euclidian geometry:

Lemma 1. A set of points is a piece of an $\alpha$-thick digital line if and only if these points can be covered by a strip of rational slope and of horizontal or vertical thickness strictly inferior to $\alpha$.

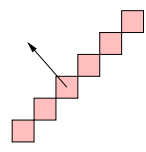

$|a|=|b|$

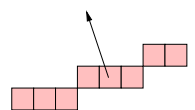

$|a|<|b|$

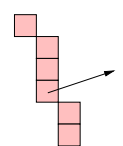

$|a|>|b|$

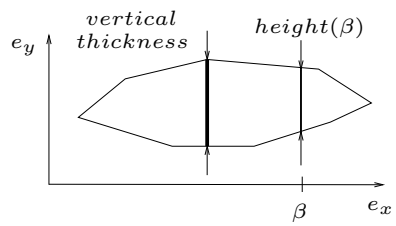

Fig. 2. Different types of digital line orientations

Fig. 3. Height and vertical thickness of a convex hull

\subsection{Thickness and Convex Hull}

In this subsection, we show that the notion of thickness (more precisely the thickness of the convex hull of the input points) plays an important role in the recognition problem.

Definition 1. The height at abscissa $\beta$ of a convex set $C$ is defined to be the length of the segment resulting from the intersection of $C$ with the vertical line $x=\beta$. We call the vertical thickness of $C$ the maximum reached by height $(x)$ (see Fig. 3). The width and the horizontal thickness are symmetrically defined.

Lemma 2. A convex polygon $N$ has a vertical thickness strictly less than $\alpha$ iff there exists a strip of vertical thickness strictly less than $\alpha$ that covers $N$.

- Proof: let $x$ denote the abscissa which corresponds to the maximum height of $N$. The upper and lower border of $N$ at abscissa $x$ can be linked to either a vertex or an edge. We can consider three different configurations:

1. edge-edge: this case only appears if both edges are parallel (see Fig. 4, b). If this were not the case, it would exist a greater value for the vertical thickness of $N$ (see Fig. 4, a). As $N$ is convex, it is included in the strip defined by these two edges. So $N$ can be covered by a strip of correct thickness.

2. edge-vertex: as the maximum is achieved at this abscissa, the line passing through this vertex and parallel to this edge is tangent to $N$ (see Fig. 4. c). We use this line and the previous edge to build a valid strip. 


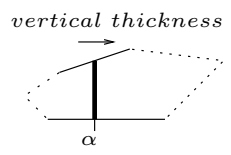

(a)

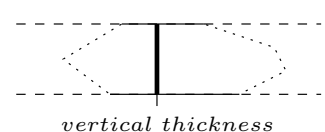

(b)

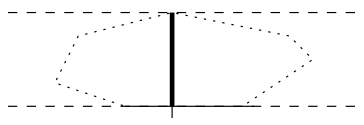

vertical thickness

(c)
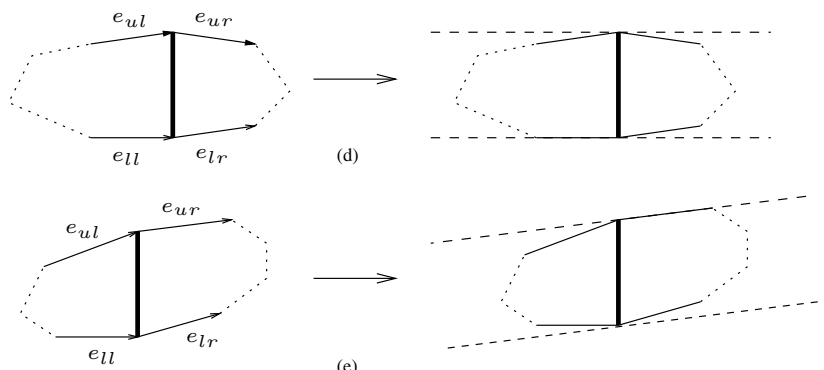

(e)

Fig. 4. The different configurations of the vertical thickness location

3. vertex-vertex: this case is a bit more complicated. Let $e_{u l}, e_{u r}, e_{l l}$ and $e_{l r}$ denote the emerging edges from these two vertices. As the polygon is convex, we have slope $_{e_{u r}} \leq$ slope $_{e_{u l}}$ and slope $_{e_{l r}} \geq$ slope $_{e_{l l}}$. The maximum height reached at this abscissa implies slope $_{e_{u l}} \geq$ slope $_{e_{l l}}$ and slope $_{e_{u r}} \leq$ slope $_{e_{l r}}$. If we choose $e_{l l}$ as a border of our strip, $e_{u l}$ and $e_{l r}$ lies inside. Assume that slope $_{e_{u r}} \leq$ slope $_{e_{l l}}$. In this case (see Fig. 4,d) $N$ is included in a valid strip. In the opposite case where slope $_{e_{u r}}>$ slope $_{e_{l l}}$ (see Fig. 4.e), this edge can not be chosen as a border for our strip. Hopefully, $e_{u r}$ is a correct choice. Indeed, we have slope $_{e_{u r}} \leq$ slope $_{e_{u l}}$ and slope $_{e_{u r}} \leq$ slope $_{e_{l r}}$ by assumptions; as slope $_{e_{u r}}>$ slope $_{e_{l l}}$ this finally implies that $N$ is included in a strip of vertical thickness strictly less than $\alpha$.

Using lemmas 1 and 22 we finally obtain the next property which links the thickness of a convex hull to the $\alpha$-thick digital line recognition problem:

Property 1. A set of points is a piece of an $\alpha$-thick digital line if and only if its convex hull has an horizontal or vertical thickness strictly inferior to $\alpha$.

\subsection{The Importance of Convex Hull}

A convex set $C$ has a vertical thickness strictly less than $\alpha$ if and only if $C$ and its translation by $\alpha . e_{y}$ have an empty intersection. Consider the case where a point $P$ is inserted on the right of $C$ (see Fig. 5. a). Let $u$ (resp. $l$ ) denote the point lying on the upper (resp. lower) border through which a supporting line (a line passing through $P$ and tangent to $C$ ) passes. If the triangle $u l P$ intersects $C+\alpha \cdot e_{y}$, then the vertical thickness of the new convex set is greater or equal to $\alpha$. Conversely, if the intersection is empty, the vertical thickness of $u l P$ is strictly less than $\alpha$, so the intersection between $u l P$ and $u l P+\alpha \cdot e_{y}$ is empty. It follows: 
(a) $u l P$ does not intersect $C H+e_{y}$

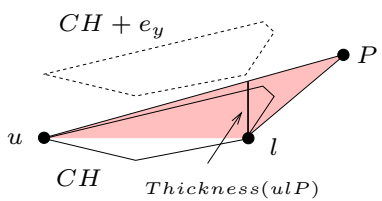

(b) area delimited by the four critical supporting lines

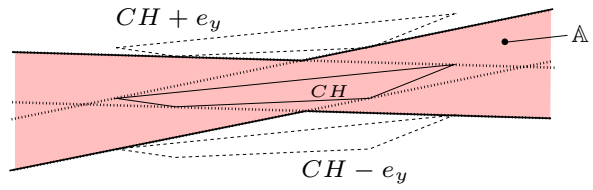

(c) When a point $P$ lies in $A, u l P$ can not intersect $C H+e_{y}$

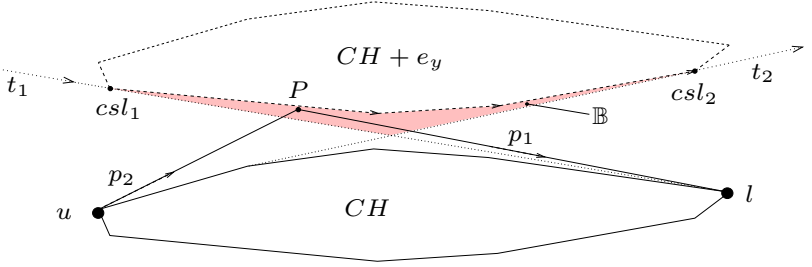

(d) Representation of $\mathbb{D}$

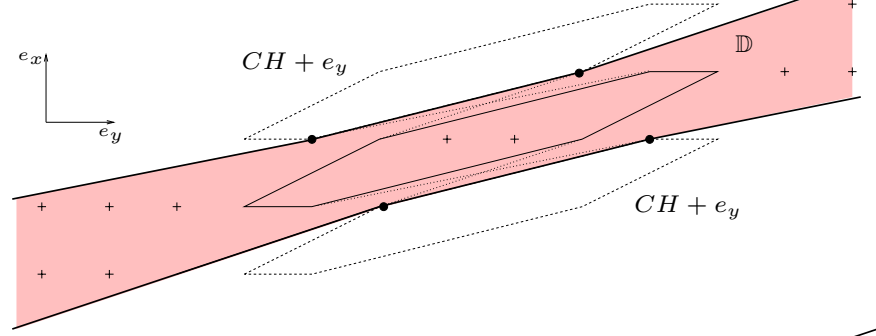

(e) Example 1 - a piece of a naive digital line

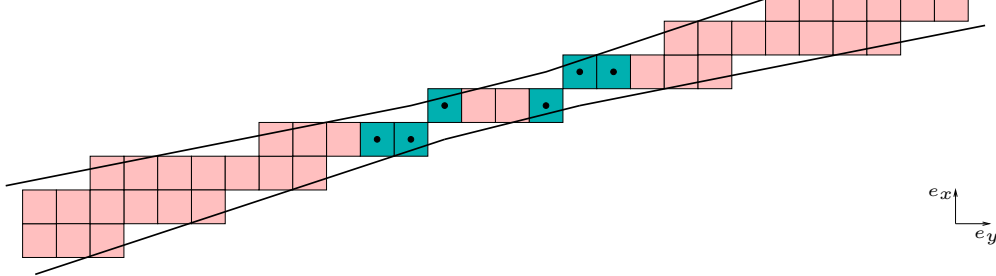

(f) Example 2 - a piece of an $\alpha$-thick digital line with $\alpha=2.5$

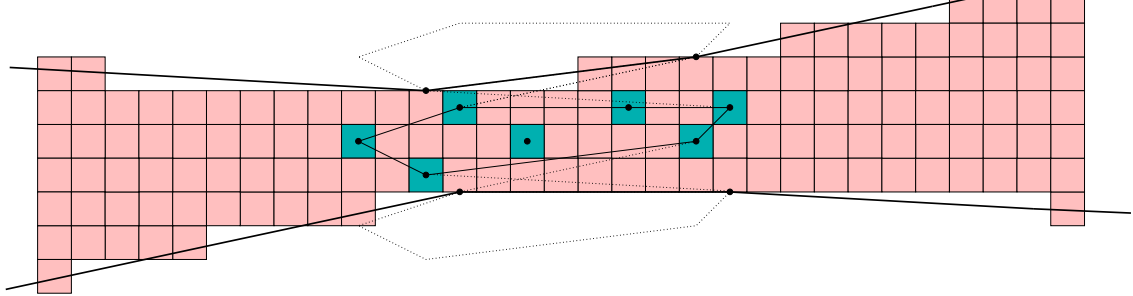

Fig. 5. Domain of the points that maintain an $\alpha$-thick digital line 
Property 2. We can insert a point to a convex set $C$ while maintaining its vertical thickness strictly below $\alpha$ if and only if the two supporting lines do not intersect $C+\alpha . e_{y}$ and $C-\alpha . e_{y}$.

For the convex hull $\mathrm{CH}$ of a given set of points, we want to determine the domain $\mathbb{D}$ of the points that can be inserted while preserving the vertical thickness of $C H$ strictly below $\alpha$. We call critical supporting lines (CSL) between two convex objects $C_{1}$ and $C_{2}$ the two lines tangent to both convex objects such that $C_{1}$ and $C_{2}$ lie on opposite sides of each line. We show that the area $\mathbb{A}($ see $[5, \mathrm{~b})$ delimited by the four CSL between $C H+\alpha \cdot e_{y}$ and $C H$ and between $C H$ and $C H-\alpha . e_{y}$ plays an important role. Indeed, according to the previous property, when a point lying in $\mathbb{A}$ is inserted the new vertical thickness is always valid. We now examine the area $\mathbb{B}$ above the two CSL $t_{1}$ and $t_{2}$ and under $C H+e_{y}$ (see Fig. 5.c). Let $c s l_{1}$ and $c s l_{2}$ denote the vertices of $C H+e_{y}$ that support $t_{1}$ and $t_{2}$. We show that when a point $P$ lying in $\mathbb{B}$ is inserted, the associated supporting segments $p_{1}$ and $p_{2}$ between $P$ and $C H$ do not intersect $C H+e_{y}$. By assumptions, $P$ is above $t_{1}$ and $t_{2}$. As $p_{1}$ (resp. $p_{2}$ ) is tangent to $C H$, it must cross $t_{1}$ (resp. $t_{2}$ ). So slope $p_{1} \leq$ slope $_{t_{1}}$ and slope $_{p_{2}} \geq$ slope $_{t_{2}}$. By convexity properties, the slopes of all the edges of $C H$ between $c s l_{1}$ and $c l_{2}$ are ranging from the slope of $t_{1}$ to the slope of $t_{2}$. Thus $p_{1}$ and $p_{2}$ can not cross $C H+e_{y}$. Finally, the upper border of $\mathbb{D}$ is delimited by the CSL lines emerging from $c s l_{1}$ and $\mathrm{csl}_{2}$ and the edges of $\mathrm{CH}+e_{y}$ located between these two vertices (see Fig. [5]d). We symmetrically define the lower border of $\mathbb{D}$. We present this result for a naive digital line (see Fig. 5]e) and an $\alpha$-thick digital line with $\alpha=2.5$ (see Fig. 5.f). The CSL can be computed in optimal linear time with the rotating calipers approach [Tou83].

\section{Algorithm Design}

\subsection{Computing the Vertical Thickness of a Convex Hull}

We recall that our definition of thickness is different from the one usually used in Computational Geometry. In this field, the thickness is linked to the strip of minimal width (computed with the $L_{2}$-norm) that encloses all the points (see Fig. 6). The rotating caliper algorithm [HoT88] can be used to compute this value in optimal linear time in the number of vertices. In the same way, we can determine the vertical thickness in linear time by using a double-traversal of the upper and lower borders of the convex hull. We set up a new approach based on convexity properties. Let $f(x)$ and $g(x)$ denote the functions defined by the upper and lower borders of the convex hull (see Fig. 7). The vertical thickness is always linked to a hull vertex (the edge-edge case is always associated to a vertexedge or vertex-vertex case). So we only have to check the value of height $(x)$ at the abscissas of the hull vertices. Notice that $f$ is concave and $g$ is convex, so the difference height $(x)=f(x)-g(x)$ is a concave function and we can apply a binary search to find its optimum. For example, suppose we have $k$ vertices on $U$ the upper border of the hull. We compute the value height $\left(U_{k / 2}\right)$ and 


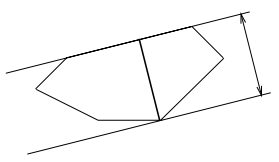

Fig. 6. The common thickness definition
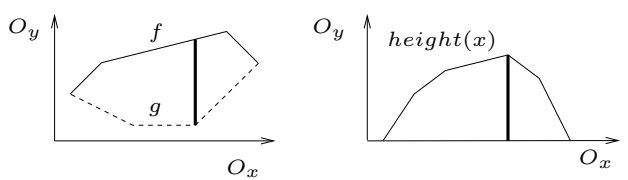

Fig. 7. The vertical thickness

height $\left(U_{k / 2+1}\right)$. By another binary search on the lower border, we determine which vertices are faced to $U_{k / 2}$ and $U_{k / 2+1}$ in $O(\log n)$ time. Suppose that height $\left(U_{k / 2}\right)<\operatorname{height}\left(U_{k / 2+1}\right)$. Then by convexity property all the vertices of indices less than $k / 2$ can not define the optimum. So we are able to reject one half of the upper vertices. We perform in the same way for the vertices of the lower hull. So we have removed at least one half of all the vertices in $O(\log n)$ time. By continuing the binary search on height $(x)$, we finally obtain the vertical thickness in $O\left(\log ^{2} n\right)$ time.

\subsection{The Incremental Approach}

This is an on-line version where no information about insertions are known in advance. At each iteration, thickness and covering strip are computed. When a new point is inserted, we compute the resulting convex hull using classical incremental convex hull algorithm [PS85,BKS95] in $O(\log n)$ time. When the point lies inside the current strip, the thickness is unchanged. When the point lies outside, we have to compute the new thickness. Using the previous method, we would obtain an $O\left(n \log ^{2} n\right)$ algorithm, but we improve the global complexity by simplifying the binary search. We only consider the case where the point is inserted on the right of the hull (see Fig. 8, a), others cases are similar (see Fig. $8 \mathrm{~b}$ ). The light grey area corresponds to a triangle; its thickness can be computed in constant time. The other area is delimited by a piece of the upper border and a tangent. Thus, all the vertices of this area are linked to the same segment and we can perform a simple binary search to find in $O(\log n)$ time the maximum height of this area. Finally, we can update the vertical thickness in $O(\log n)$ time per insertion.

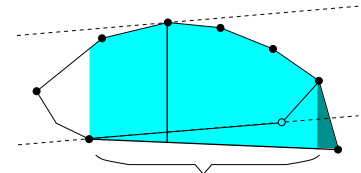

(a)

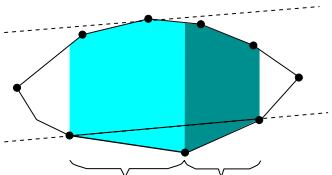

(b)

Fig. 8. Computing the new thickness in the incremental method 


\subsection{A Special Case}

We often know in real applications that points are inserted in the same direction. Previous algorithms [DR95] take advantage of this in order to obtain a linear time complexity. We present an elementary implementation designed for this special case. First, we recall that the incremental convex hull under this assumption can be processed in $O(n)$ time using classical methods [Mlk87]. We choose to insert points by increasing abscissa (see Fig. 9). Notice that insertions on the right of the convex hull shifts the vertical thickness to the right. Let $\beta$ denote the abscissa where the previous vertical thickness was located. The dimmed area is delimited by the line $x=\beta$, a border of the previous strip and a tangent. It covers the left part of the new convex hull. In this triangle, all values of height $(x)$ are less or equal to height $(\beta)$. So the vertical thickness must be located on the right of this area. We do not use a binary search anymore. We just traverse each vertex on the right up to the maximal height. Each trial has a $O(1)$ cost, and the total number of trials is bounded by the total amount of inserted points. So the incremental recognition of a digital line of thickness $d$ can be computed in $O(n)$ time if we insert points in a given order.

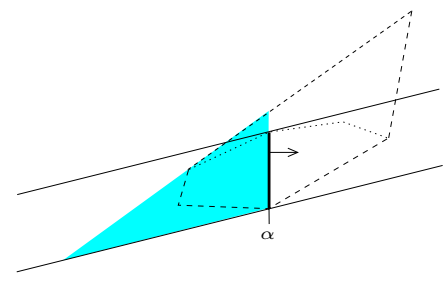

Fig. 9. Insertion on the right shifts vertical thickness to the right

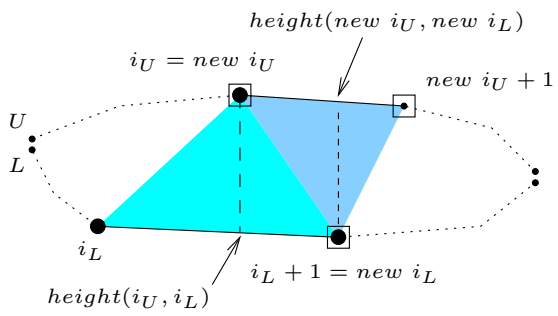

Fig. 10. Notations of the algorithm

\subsection{Pseudo-Code}

We present (see Fig. 13) the main function used to compute the vertical thickness of a convex hull: UPDATE_VERTICAL_THICKNESS. The hull will consist of two lists $U$ and $L$ corresponding to its upper and lower borders. Let $n_{U}$ and $n_{L}$ denote the size of $U$ and $L$ respectively. We call $i_{U}$ and $i_{L}$ the indices of the couple of vertices lying on $U$ and $L$ respectively that define the vertical thickness. When the case edge-vertex appears, we select the left vertex of the edge. The other vertex is necessarily the one of $U_{i_{U}+1}$ and $L_{i_{L}+1}$ with minimum abscissa (function HEIGHT). We call new $i_{U}$ and new $i_{L}$ (see Fig. 10) the indices of vertices that are associated with the next discontinuity of height $(x)$. The implementation presented here processes points entered from left to right (the insertion order in the vertical direction is unimportant). The same function can be used in the three others directions by symmetrizing the $(x, y)$ coordinates. 


\subsection{Complexity of the Algorithms}

We may use dynamic convex hull (incremental and decremental) that induces an $O\left(n \log ^{2} n\right)$ overhead [PS85]. We recall the complexities of our methods:

\begin{tabular}{|c|c|c|c|c|}
\hline case & convex hull & incremental & in one direction & dynamic \\
\hline thickness computation & $O\left(\log ^{2} n\right)$ & $O(n \log n)$ & $O(n)$ & $O\left(n \log ^{2} n\right)$ \\
\hline
\end{tabular}

\subsection{Applications of $\alpha$-Thick Lines}

We are able to convert any set of points into a polygonal chain of digital line segments, even if no assumptions on connectedness can be done and no information about thickness is known a priori. We present in Fig. 11 a first test on a low quality digitization. We may adjust the thickness in order to obtain a compromise between the number of segments and the conversion quality.

Pixel Traversal Order. First, we have to choose the pixel order that will be used in our recognition process. Pixels are stored in a data structure $L$ that allows efficient range searching. Suppose we have a function STARTING_POINT that arbitrary selects a starting pixel. This point represents the first level and it can be removed from $L$. To fill the next level we only have to select pixels that are in the neighborhood of the pixels in the current level (using a particular distance function). When no pixels are found, we widen the current neighborhood until we find one or more points. When a maximal thickness $\delta$ is allowed for the recognition, it is judicious to limit the maximal neighborhood to a width $\delta$. Thus, the holes of at most $\delta$ pixels can be filled. The pixels of the same level are arbitrary ordered. We recall the entire process in the following algorithm and present an example in figure 12 .

The Overall Method. We process pixels one after the other relative to the predefined order. When the thickness of the current digital segment exceeds the given threshold $\delta$, we backtrack to the previous pixel and select the corresponding digital segment and the associated thickness (that may be between 0 and $\delta$ ). When we want to obtain a polygonal chain, we may insert the last pixel of the previous digital segment in the next one. The method presented here is quite elementary and is based on a greedy approach. Thus the result is completely dependent of the chosen pixel traversal order and no uniqueness can be obtained.

Open Problems. When we have worked with digital segments, no considerations have been done about the quality of the jonction between two consecutive digital segments. In fact, pieces of them can cover themselves at the jonction. Optimizations relatively to the number of digital segments and to the visual quality remain to solve. Thus many questions are left untreated and their management would require an improved method that goes beyond the scope of this paper. 


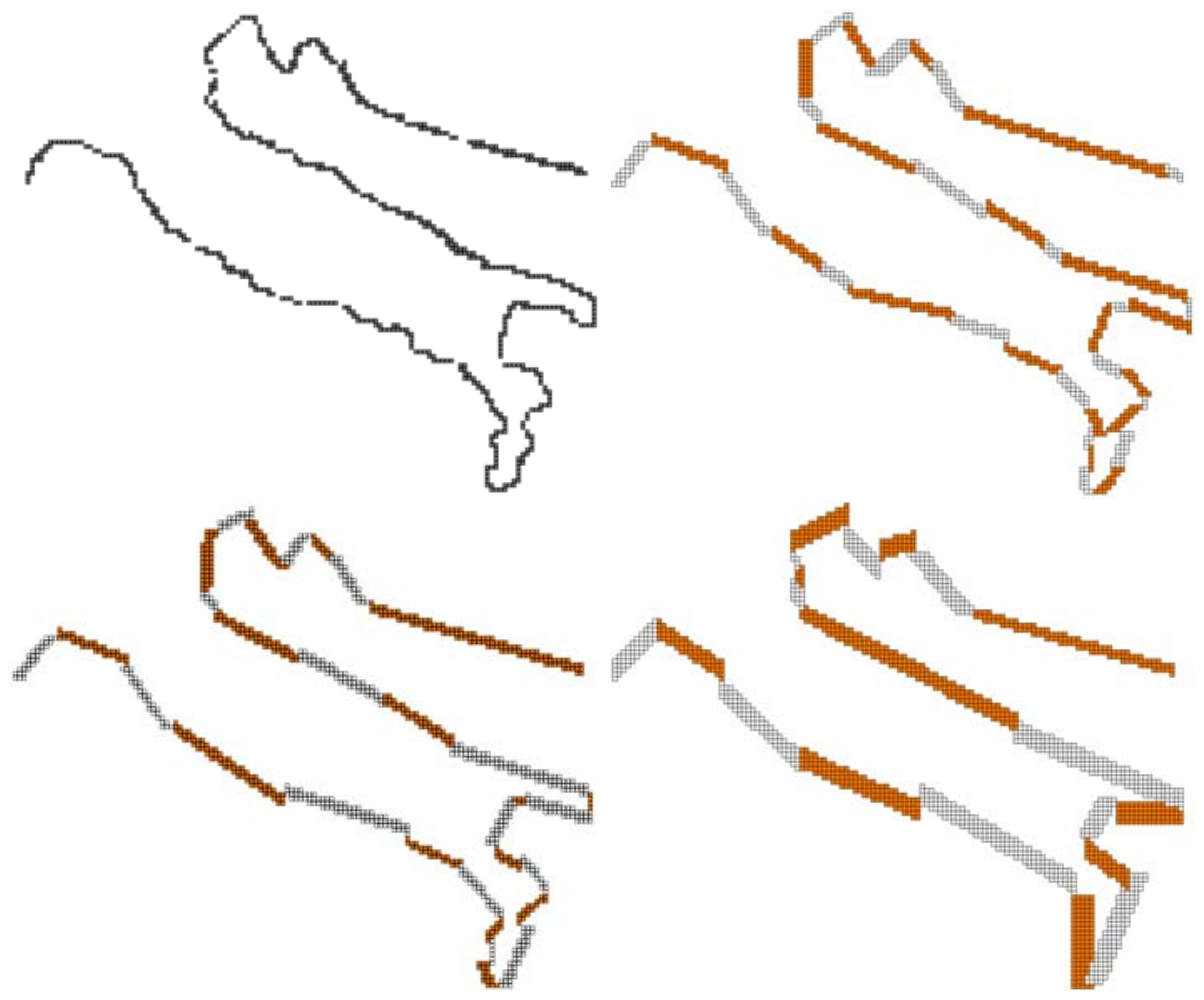

Fig. 11. A low quality digitization of the Italian coast converted using $\alpha$-thick digital segments $(\alpha=2.5,3$ and 4.5). We successively obtain 37, 30 and 20 digital segments

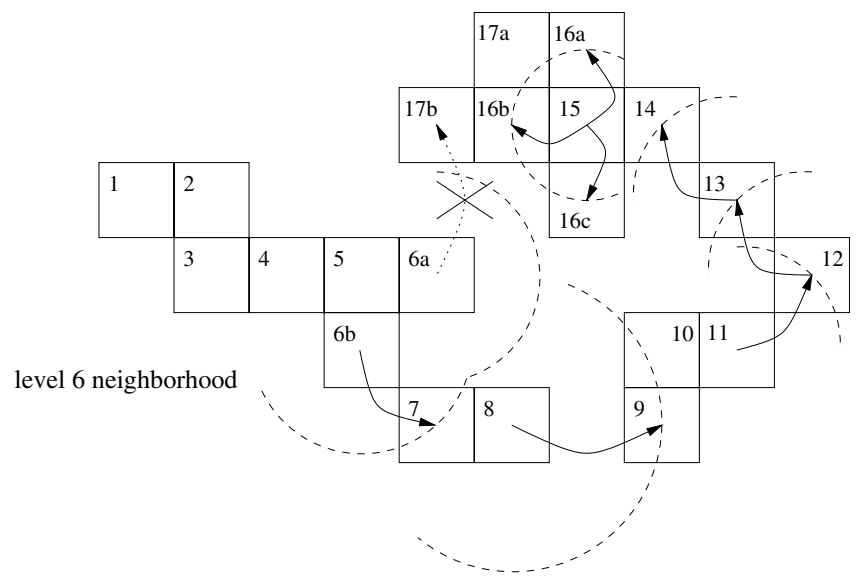

Fig. 12. Computing the pixel order traversal 

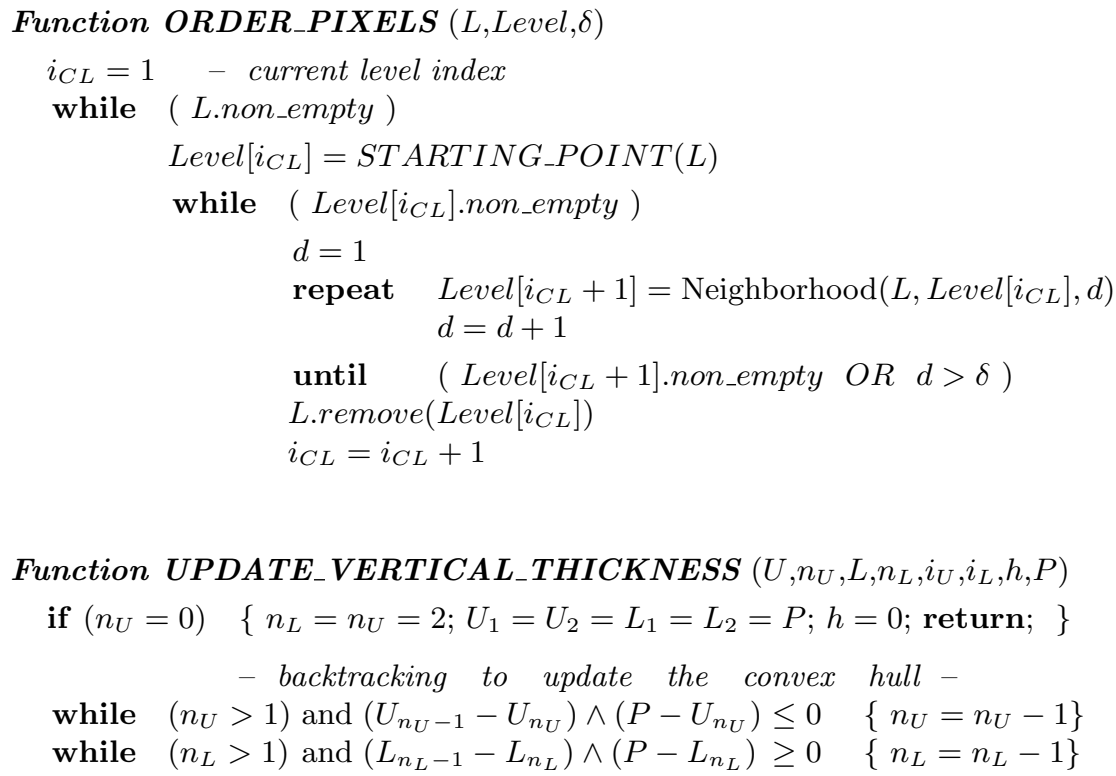

- when a vertex that defines the previous thickness is removed if $\left(i_{U}>n_{U}\right) \quad\left\{i_{U}=n_{U}\right\}$ if $\left(i_{L}>n_{L}\right) \quad\left\{i_{L}=n_{L}\right\}$

$n_{L}=n_{L}+1 ; \quad L_{n_{L}}=P$ - insertion of the new point $P$ $n_{U}=n_{U}+1 ; \quad U_{n_{U}}=P$ $h=H E I G H T\left(U, L, i_{U}, i_{L}\right)$;

- traverse the hull while the height increases while $\left(i_{L} \neq n_{L}-1\right)$ or $\left(i_{U} \neq n_{U}-1\right)$

Fig. 13. The core function of the recognition method based on thickness computation

\section{Conclusion and Future Works}

We introduce a new digital line recognition method based on the vertical and horizontal thickness. Our method is well adapted to recognize any set of points 
where no assumptions on connectedness can be done. The internal machinery uses classical convex hull algorithms.

The knowledge of the convex hull of the current set of points allows to increase the thickness computation efficiency, to make easier any implementation in the two-dimensional case, to determine the points that can be inserted while preserving a valid digital line, to obtain an incremental or dynamic method and to extend our approach to points with real coordinates. We do not deal with the strategy used to order points during the recognition. At each step, the thickness is computed and we may suppose that the associated normal vector is close to the local curvature. Thus, we may take advantage of this information in order to enhance the overall conversion quality. Other criteria may be inserted like density. Indeed, we may consider the ratio between the number of points inside the hull and the hull surface. This would be an automatic criterion that could replace the usual predefined thickness threshold. Such strategies combined with the dynamic approach could lead to a new generation of adaptive algorithms...

The source code in $\mathrm{C}++$ of our method as well as a basic geometry kernel, some examples and some tests can be downloaded from the web page of the author: www.esiee.fr/ buzerl/DG/.

\section{References}

[BKS95] M. de Berg, M. van Kreveld, and S. Schirra. A new approach to subdivision simplification. In Proc. of Auto-Carto 12, pages 79-88, 1995.

[Buz02] L. Buzer. Reconnaissance des plans discrets - Simplification polygonale. Thèse, Université d'Auvergne, Clermont-Ferrand, 2002.

[Buz03] L. Buzer. A linear incremental algorithm for naive and standard digital lines and planes recognition, Graphical Models, 65(1-3), pp. 61-76, 2003.

[DR95] I. Debled-Rennesson, J.-P. Reveillès. A linear algorithm for segmentation of digital curves. International Journal of Pattern Recognition and Artificial Intelligence, Volume 9, N. 6, dec. 1995.

[Hot88] M. E. Houle and G. T. Toussaint. Computing the width of a set. IEEE Trans. Pattern Anal. Mach. Intell., PAMI-10, 761-765, 1988.

[Mlk87] A.A. Melkman. On-line construction of the convex hull of a simple polyline. Information Processing Letters, 25:11-12, 1987.

[PS85] F.P. Preparata, M.I. Shamos. Computational Geometry. Springer-Verlag, New-York, 1985.

[Rev91] J.P. Reveillès. Géometrie discrète, calculs en nombre entiers et algorithmique. Thèse d'état, Université Louis Pasteur, Strasbourg, 1991.

[Tou83] G.T. Toussaint. Solving geometric problems with the rotating calipers. Proceedings of IEEE MELECON'83, Athens, Greece, May 1983. 